ESAIM: PROCEEDINGS, September 2005, Vol.14, 48-62

Eric Cancès \& Jean-Frédéric Gerbeau, Editors

DOI: $10.1051 /$ proc:2005005

\title{
AN EMBEDDED SURFACE METHOD FOR VALVE SIMULATION. APPLICATION TO STENOTIC AORTIC VALVE ESTIMATION*
}

\author{
Paola Causin ${ }^{1}$, Nuno Diniz dos Santos ${ }^{2}$, Jean-Frédéric Gerbeau ${ }^{3}$, \\ Carine Guivier ${ }^{4}$ And Paul MÉtier ${ }^{5}$
}

\begin{abstract}
This work deals with the modelling and the simulation of a moving rigid valve in a blood flow. We consider a fluid-structure coupling between an unsteady incompressible Navier-Stokes system for the fluid, and an ODE for the structure with a rotation angle as only degree of freedom. The kinematic constraint on the fluid-structure interface is enforced via a Lagrange multiplier. A fictitious domain approach allows us to use a fixed grid for the fluid domain, and also to easily consider large valve displacements. We present numerical results and we discuss clinical indexes used to characterize aortic valve stenosis.
\end{abstract}

Résumé. Nous présentons un travail de modélisation et simulation d'une valve rigide dans un écoulement sanguin. Nous considérons un couplage fluide-structure où l'écoulement sanguin est régi par le système de Navier-Stokes instationnaire incompressible, tandis que la dynamique de la valve est ramenée à une EDO correspondant à une rotation. La contrainte cinématique à l'interface fluidestructure est imposée par un multiplicateur de Lagrange. Une approche de type domaine fictif nous permet d'adopter un maillage fixe pour le domaine fluide et de traiter aisément les grands déplacements de la valve. Nous présentons des simulations numériques et nous discutons d'indices cliniques utilisés pour caractériser des sténoses aortiques.

\section{INTRODUCTION}

The aortic valve is charged to let oxygenated blood flow from the heart left ventricle into the aorta (see Fig. 1). The aortic valve is composed of three leaflets, or flaps of connective tissue, that passively move apart or mate together in response to the pressure gradients imposed by the blood pumped by the heart.

Among the pathologies that may interest the aortic valve, aortic stenosis affects approximately 5 out of every 10,000 people in the Western countries (see for example [15]). Stenosis is an abnormal narrowing of the valve, due to congenital malformation or calcification, which prevents the valve from opening properly. Once stenosis develops, high overpressure builds up in the left ventricle, which can seriously injure the heart.

\footnotetext{
* This work has been partially supported by the Research Training Network "Mathematical Modelling of the Cardiovascular System" (HaeMOdel), contract HPRN-CT-2002-00270 of the European Community.

${ }^{1}$ CMCS/IACS, Ecole Polytechnique Fédérale de Lausanne, CH-1015 Lausanne, Switzerland; paola.causin@epfl.ch

2 INRIA - Projet REO - B.P.105 F-78153 Le Chesnay Cedex, France; Nuno-Miguel.Diniz-Dos-Santos@inria.fr

${ }^{3}$ INRIA - Projet REO - B.P.105 F-78153 Le Chesnay Cedex, France; Jean-Frederic.Gerbeau@inria.fr

${ }^{4}$ IRPHE UMR 6594, Equipe de Biomécanique Cardiovasculaire, Institut Méditerranéen de Technologie, F-13451 Marseille cedex

20, France; guivier.carine@esm2.imt-mrs.fr

5 Commissariat à l'Énergie Atomique (CEA), DEN/DM2S/SFME/LTMF Saclay, F-91191 Gif sur Yvette Cedex, France; pmetier@cea.fr
}

(c) EDP Sciences, SMAI 2005 


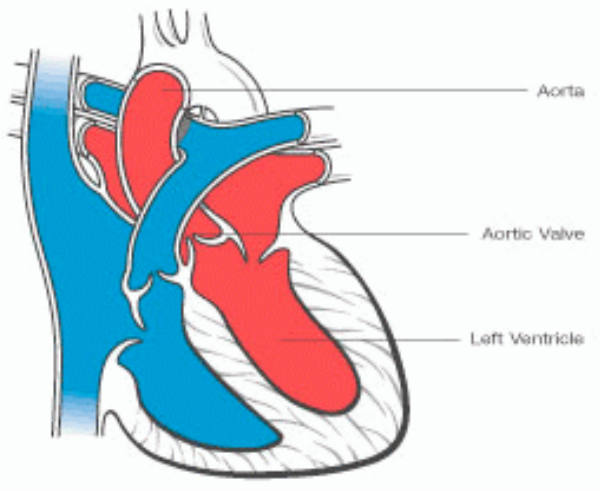

FiguRE 1. Halfplane anatomical sketch of the aortic valve and the heart (from the American Heart Association).

In general, when dealing with the establishment of guidelines for the diagnosis and treatment of a certain pathology, the relevant steps are:

(i) identify the physical causes of certain symptoms: in the case of the aortic stenosis, the narrowing of the valve leading to reduced blood flow and pressure buildup inside the heart that can produce chest pain, fainting, loss of consciousness, rapid or irregular heartbeats;

(ii) identify appropriate synthetic indexes evaluable with diagnostic tools (in case of suspect aortic stenosis, Doppler and cathetherization are generally used) that provide a quantitative measure of the above physical phenomena and can drive the clinical diagnosis and the treatment of the patient;

(iii) evaluate the validity of these indexes with respect to their adherence to the actual outcome of the pathology.

It seems out of reach for the moment to purely use numerical simulations to move from (i) to (ii) and statistical studies seem to be the tool the most adapted to point iii). Nevertheless, numerical simulations might play an important role in evaluating the foundation of a specific clinical index that is supposed to measure a relevant physical quantity (step (ii)). Indeed, in general very strong assumptions and several simplifications are done to derive practical indicators. Numerical simulations may thus help to assess the correspondence of a certain index with respect to the actual complex physical phenomenon.

In the case of the aortic valve stenosis, it is generally accepted that a relevant synthetic index is the so-called Effective Orifice Area (EOA), which is the minimal cross-sectional area of the flow jet, attained downstream the valve. The EOA can be measured either by Doppler or by catheterization. It has been recently advocated in [10] that the energy loss through the valve could be a better indicator of the effect of the stenosis on the overall hemodynamic field. The authors propose an energy loss index that can be derived from non-invasive measurements. Validation of this index has been carried out in [10] by performing statistical studies that show how the energy loss index has a more significant correlation with the mortality and morbidity of several patients with respect to other indexes as for example the EOA.

Concentrating on the energy loss, numerical simulations can be used in the sense advocated above, that is to assess whether this indicator reflects the "true" energy loss computed by the mathematical model. In this preliminary work, we propose a numerical framework that allows for simulating a simplified aortic valve affected by different degrees of stenosis and we exhibit the influence of the stenosis on the energetic dissipation.

The following of this work is divided into four parts: we first introduce the mathematical model in Sect. 2; in Sect. 3 we propose a numerical method based on the fictitious domain/Lagrange multiplier approach [12]; the problem of clinical assessment is presented in Sect. 4; we eventually present some numerical results in Sect. 5. 


\section{Mathematical modelling}

The spatial configuration of the aortic valve and of its surroundings parts has a complex 3D geometry. Nevertheless, in this preliminary work, we restrict our study to a $2 \mathrm{D}$ domain $\Omega$, sketched in Fig. 2: the valve is represented by two flaps, and the problem is symmetric with respect to the central axis. We consider that each

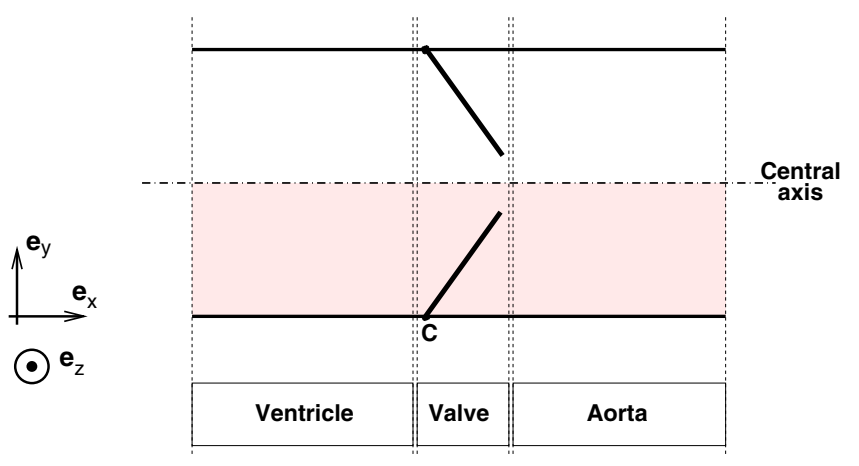

Figure 2. Bi-dimensional scheme of the area of interest.

leaflet of the valve is rigid which is of course not realistic for biological valves but which is actually the case for some prosthetic aortic valves. This choice makes the structure equation pretty simple. However, the proposed algorithm does not pose limits in terms of the structure model, that can be elastic as well. The contact problem, and therefore the complete closure or complete opening of the valve, will not be considered in this article.

Let $\Omega$ be the $2 \mathrm{D}$ bounded domain depicted in Fig. 3 and $\left(\boldsymbol{e}_{x}, \boldsymbol{e}_{y}, \boldsymbol{e}_{z}\right)$ the $\mathbb{R}^{3}$ basis indicated on the figure. We adopt the following standard convention: let $\boldsymbol{u}=\left(u_{x}, u_{y}\right)$ and $\boldsymbol{v}=\left(v_{x}, v_{y}\right)$ be two vectors of $\mathbb{R}^{2}$, then $\boldsymbol{e}_{\boldsymbol{z}} \times \boldsymbol{u}$ is the $\mathbb{R}^{2}$ vector defined by $\left(-u_{y}, u_{x}\right)$ and $\boldsymbol{u} \times \boldsymbol{v}$ is the scalar defined by $u_{x} v_{y}-u_{y} v_{x}$.

\subsection{Structure model}

We denote by $\Sigma(t)$ the $1 \mathrm{D}$ segment included in $\Omega$ which represents the structure domain at time $t \in(0, T)$, $T>0$. The only degree of freedom of the valve is its rotation angle $\theta$ about a fixed axis whose trace on the plane is a point $\mathbf{C}$. The equation governing the angle is therefore a single ordinary differential equation, that expresses the conservation of the angular momentum

$$
J \frac{d^{2} \theta}{d t^{2}}=\mathcal{M}_{\Sigma}
$$

$J$ being the inertia momentum of the leaflet and $\mathcal{M}_{\Sigma}$ the external momentum applied to the leaflet which comes uniquely from the hydrodynamics stress.

\subsection{Fluid model}

The fluid domain is defined by $\Omega_{F}(t)=\Omega \backslash \Sigma(t)$. We consider in $\Omega_{F}(t)$ an homogeneous, viscous, and incompressible fluid governed by the unsteady incompressible Navier-Stokes equations

$$
\left\{\begin{array}{rllll}
\rho\left(\frac{\partial \boldsymbol{u}}{\partial t}+\boldsymbol{u} \cdot \nabla \boldsymbol{u}\right)-\eta \Delta \boldsymbol{u}+\nabla p & =0 & \text { in } & \Omega_{F}(t) \times(0, T), \\
\operatorname{div} \boldsymbol{u} & = & 0 & \text { in } & \Omega_{F}(t) \times(0, T),
\end{array}\right.
$$

where $\boldsymbol{u}$ is the fluid velocity, $p$ the pressure, $\rho$ the fluid density, $\eta$ the dynamic viscosity. The Cauchy stress is denoted by

$$
\boldsymbol{\sigma}=-p \mathbf{I}+2 \eta \mathbf{D}(\boldsymbol{u}),
$$


where $\mathbf{I}$ is the identity tensor and $\mathbf{D}(\boldsymbol{u})$ is the strain rate $\left(\nabla \boldsymbol{u}+\nabla \boldsymbol{u}^{T}\right) / 2$. We denote by $\boldsymbol{n}$ the outward normal on $\partial \Omega_{F}(t)$. No-slip boundary conditions are imposed on the wall $\Gamma_{0}$, symmetry boundary conditions on the central axis $\Gamma_{c}$, and the value of the total stress is prescribed at the inlet $\Gamma_{\text {in }}$ and the outlet $\Gamma_{\text {out }}($ Fig. 3$)$ :

$$
\left\{\begin{aligned}
\boldsymbol{\sigma} \cdot \boldsymbol{n}(\cdot, t) & =-p_{\text {in }}(\cdot, t) \boldsymbol{n} & & \text { on } & \Gamma_{\text {in }} \times(0, T), \\
\boldsymbol{\sigma} \cdot \boldsymbol{n} & =0 & & \text { on } & \Gamma_{\text {out }} \times(0, T),
\end{aligned}\right.
$$

where $p_{\text {in }}$ is a given function. An alternative choice to represent the injection of fluid would be to impose a velocity profile over $\Gamma_{\text {in }}$ or a flux (see e.g. [8] or [7]). The system is also completed with suitable initial conditions.

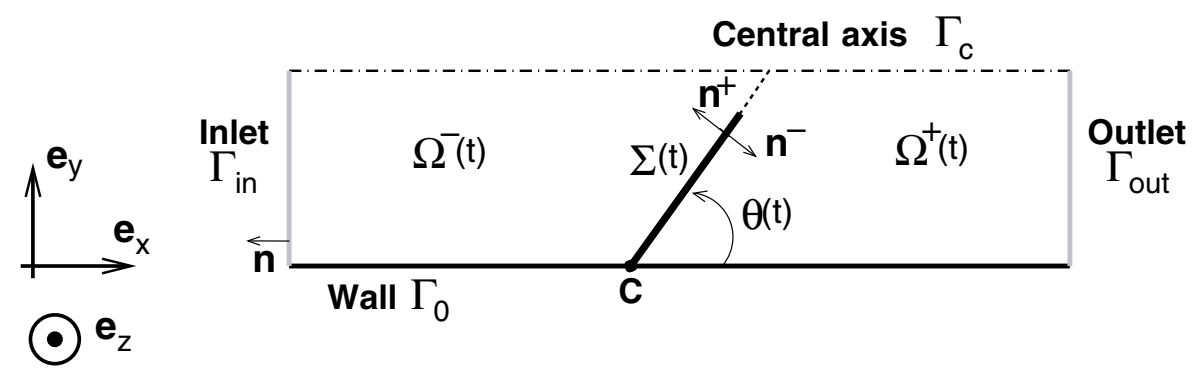

FiguRE 3. Bi-dimensional configuration.

The valve $\Sigma(t)$ defines a natural partition of $\Omega_{F}(t)$ into two subdomains $\Omega^{-}(t)$ and $\Omega^{+}(t)$ (see Fig. 3 ). On $\Sigma(t)$, we define $\boldsymbol{n}^{+}$(resp. $\boldsymbol{n}^{-}$) as the outgoing normal on $\partial \Omega^{+}$(resp. $\partial \Omega^{-}$), and:

$$
\boldsymbol{f}_{\Sigma}=-\left(\boldsymbol{\sigma}^{+} \cdot \boldsymbol{n}^{+}+\boldsymbol{\sigma}^{-} \cdot \boldsymbol{n}^{-}\right)
$$

where $\boldsymbol{\sigma}^{+}(\mathbf{x})$ (resp. $\left.\boldsymbol{\sigma}^{-}(\mathbf{x})\right)$, for $\mathbf{x} \in \Sigma(t)$, is the limit of $\boldsymbol{\sigma}\left(\mathbf{x}-\varepsilon \boldsymbol{n}^{+}\right.$) as $\varepsilon$ goes to $0^{+}$(resp. $0^{-}$).

\subsection{Fluid-structure interaction}

The fluid-structure coupling takes place on the fluid-structure interface. In the configuration at hand, the structure being immersed and having one dimension less than the fluid, the interface coincides with the structure domain $\Sigma(t)$. The continuity of the velocities on the interface reads:

$$
\boldsymbol{u}(\mathbf{x}(t), t)=\omega \mathbf{e}_{z} \times \mathbf{C x}(t)
$$

for $\mathbf{x}(t) \in \Sigma(t), t \in(0, T)$ and $\mathbf{C}$ the center of the rotation (see Fig. 3); we have also defined the angular velocity:

$$
\omega=\frac{d \theta}{d t}
$$

The momentum on the structure is given by:

$$
\mathcal{M}_{\Sigma}(t)=\int_{\Sigma(t)} \mathbf{C x} \times \boldsymbol{f}_{\Sigma} d l(\mathbf{x}) \quad t \in(0, T),
$$

where $\boldsymbol{f}_{\Sigma}$ is defined by $(3)$. 


\subsection{Energy balance of the coupled system}

For the structure part of the system, multiplying (1) by $\frac{d \theta}{d t} \mathbf{e}_{z}$, and using (5), we obtain:

$$
\frac{1}{2} J \frac{d}{d t}\left(\left|\frac{d \theta}{d t} \mathbf{e}_{z}\right|^{2}\right)=\int_{\Sigma(t)} \frac{d \theta}{d t} \mathbf{e}_{z} \cdot\left(\mathbf{C x} \times \boldsymbol{f}_{\Sigma}\right)=\int_{\Sigma(t)}\left(\frac{d \theta}{d t} \mathbf{e}_{z} \times \mathbf{C x}\right) \cdot \boldsymbol{f}_{\Sigma} .
$$

For the fluid part, multiplying the first equation of (2) by $\boldsymbol{u}$ and integrating over $\Omega_{F}(t)$ at a fixed time $t$, we have, using the free divergence of the velocity and the boundary conditions:

$$
\int_{\Omega_{F}(t)} \rho\left(\frac{\partial \boldsymbol{u}}{\partial t}+\boldsymbol{u} \cdot \nabla \boldsymbol{u}\right) \cdot \boldsymbol{u}+\int_{\Omega_{F}(t)} 2 \eta|\mathbf{D}(\boldsymbol{u})|^{2}=\int_{\Gamma_{\mathrm{in}}}\left(-p_{i n} \boldsymbol{n}\right) \cdot \boldsymbol{u}+\int_{\Sigma(t)}\left(\boldsymbol{\sigma}^{+} \cdot \boldsymbol{n}^{+}+\boldsymbol{\sigma}^{-} \cdot \boldsymbol{n}^{-}\right) \cdot \boldsymbol{u}
$$

With the definition (3) of $\boldsymbol{f}_{\Sigma}$ and the kinematic conditions (4), equations (6) and (7) yield:

$$
\frac{1}{2} \frac{d}{d t} \int_{\Omega_{F}(t)} \rho|\boldsymbol{u}|^{2}+\frac{J}{2} \frac{d\left(\omega^{2}\right)}{d t}+\int_{\Omega_{F}(t)} 2 \eta|\mathbf{D}(\boldsymbol{u})|^{2}=-\int_{\Gamma_{\mathrm{in}}} p_{i n} \boldsymbol{n} \cdot \boldsymbol{u} .
$$

The first two terms are respectively the kinetic energy of the fluid and of the structure. The only dissipative term comes from the fluid viscosity.

\section{NumericAl METHOdS}

In this section we address the numerical techniques we have considered for the discretization of the problem.

\subsection{Time discretization and coupling strategy}

In this study, the structure equation is very simple. As a consequence, it could have been implemented directly in the fluid solver. Nevertheless, we decided to keep the fluid and the structure codes independent. By doing so, more complex structures can be implemented without changing neither the fluid solver nor the coupling algorithm. Moreover, each sub-problem can be tackled with the most appropriate solver.

For the time discretization of the fluid problem, we adopt a semi-implicit Euler scheme for the fluid equation:

$$
\left\{\begin{aligned}
\rho \frac{\boldsymbol{u}^{n+1}-\boldsymbol{u}^{n}}{\delta t}+\rho \boldsymbol{u}^{n} \cdot \nabla \boldsymbol{u}^{n+1}-\eta \Delta \boldsymbol{u}^{n+1}+\nabla p^{n+1} & =0 \text { in } \Omega_{F}(t), \\
\operatorname{div} \boldsymbol{u}^{n+1} & =0 \text { in } \Omega_{F}(t),
\end{aligned}\right.
$$

and a mid-point scheme for the structure

$$
\left\{\begin{aligned}
J \frac{\omega^{n+1}-\omega^{n}}{\delta t} & =\mathcal{M}_{\Sigma}^{n+1} \\
\frac{\omega^{n+1}+\omega^{n}}{2} & =\frac{\theta^{n+1}-\theta^{n}}{\delta t}
\end{aligned}\right.
$$

and we treat implicitly the coupling conditions:

$$
\left.\boldsymbol{u}^{n+1}\right|_{\Sigma}=\boldsymbol{u}_{\Sigma}^{n+1}
$$

with

$$
\boldsymbol{u}_{\Sigma}^{n+1}(\mathbf{x})=\omega^{n+1} \mathbf{e}_{z} \times \mathbf{C x}, \quad \forall \mathbf{x} \in \Sigma^{n+1}
$$


and

$$
\mathcal{M}_{\Sigma^{n+1}}^{n+1}=\int_{\Sigma^{n+1}} \mathbf{C x} \times \boldsymbol{f}_{\Sigma}^{n+1} d l(\mathbf{x})
$$

where $\boldsymbol{f}_{\Sigma}^{n+1}$ is defined as in (3) by

$$
\boldsymbol{f}_{\Sigma}^{n+1}=-\left(\boldsymbol{\sigma}^{n+1,+} \cdot \boldsymbol{n}^{+}+\boldsymbol{\sigma}^{n+1,-} \cdot \boldsymbol{n}^{-}\right) .
$$

In order to solve the implicitly coupled fluid-structure problem, an efficient strategy must be devised. Here, a fixed point iterative procedure between problems (9)-(11) and (10)-(12) with Aitken acceleration [11,18] has been used.

\subsection{Space discretization}

Due to the iterative strategy presented above, we can focus only on the space discretization of equations (9) with the Dirichlet boundary condition (11), where $\Sigma^{n+1}$ and $\boldsymbol{u}_{\Sigma}^{n+1}$ are assumed to be given (these quantities come from the structure solver and are known from the previous fixed point iteration).

The valve movement offers to blood a varying geometrical domain. Thus, the fluid problem should be solved at each time in a different geometrical configuration. Since the displacements of the valve are very large (from close to open position, each valve leaflet sweeps approximately 90 degrees), frequent remeshing should be performed if an Arbitrary Lagrangian Eulerian approach had been chosen. This solution has been successfully applied in complex simulations (see [17] for example), nevertheless, in order to avoid remeshing and the problems connected to it, we have adopted an "embedded surface" /Lagrangian multiplier technique. With this term we indicate an approach where the fluid domain is fixed in time and overlaps also the physical region occupied by the structure immersed in it, like in the fictitious domain approaches [13]. The presence of the moving structure is recovered by a Lagrangian multiplier that augments the problem enforcing the continuity of the fluid and structure velocity fields. We refer for example to [12] for a general discussion of a Lagrange mutiplier/fictitious domain approach in the case of particles moving in a fluid and to [1] and Baaijens' group successive works for a version of this methodology applied to aortic valve simulation (for example [5]).

\subsubsection{Weak formulation of the augmented fluid problem}

Problem (9) is discretized in space with a finite element method and condition (11) is imposed via a Lagrange multiplier. We introduce the following functional spaces:

$$
\begin{aligned}
X & =\left\{\boldsymbol{v} \in\left(H^{1}(\Omega)\right)^{2}, \boldsymbol{v}=0 \text { on } \Gamma_{0}, \boldsymbol{v} \cdot \boldsymbol{n}=0 \text { on } \Gamma_{c}\right\}, \\
V & =\left\{\boldsymbol{v} \in X, \operatorname{Tr}_{\Sigma}(\boldsymbol{v})=0, \operatorname{div} \boldsymbol{v}=0\right\} \\
V\left(\boldsymbol{u}_{\Sigma}\right) & =\left\{\boldsymbol{v} \in X, \operatorname{Tr}_{\Sigma}(\boldsymbol{v})=\boldsymbol{u}_{\Sigma}, \operatorname{div} \boldsymbol{v}=0\right\}
\end{aligned}
$$

where $\operatorname{Tr}_{\Sigma}:\left(H^{1}(\Omega)\right)^{2} \longrightarrow\left(H^{1 / 2}(\Sigma)\right)^{2}$ denotes the trace operator on $\Sigma$.

We recall that $\Sigma^{n+1}$ and $\boldsymbol{u}_{\Sigma^{n+1}}^{n+1}$ are assumed to be given. A first variational formulation of the fluid problem is: find $\boldsymbol{u}^{n+1} \in V\left(\boldsymbol{u}_{\Sigma^{n+1}}^{n+1}\right)$ such that, for all $\boldsymbol{v} \in V$,

$$
\int_{\Omega} \rho \frac{\boldsymbol{u}^{n+1}-\boldsymbol{u}^{n}}{\delta t} \cdot \boldsymbol{v}+\int_{\Omega} \rho \boldsymbol{u}^{n} \cdot \nabla \boldsymbol{u}^{n+1} \cdot \boldsymbol{v}+\int_{\Omega} 2 \eta \mathbf{D}\left(\boldsymbol{u}^{n+1}\right): \mathbf{D}(\boldsymbol{v})=-\int_{\Gamma_{i n}} p_{i n} \boldsymbol{n} \cdot \boldsymbol{v} .
$$

In view of the discretization, it is much more convenient to work with space $X$. Thus, following [12], we introduce the Lagrange multiplier spaces

$$
\begin{aligned}
M & =L^{2}(\Omega), \\
\mathcal{L}^{n+1} & =\left(H^{-1 / 2}\left(\Sigma^{n+1}\right)\right)^{2},
\end{aligned}
$$


corresponding to the two constraints which define the space $V\left(\boldsymbol{u}_{\Sigma^{n+1}}^{n+1}\right)$. We then consider the following variational formulation: find $\left(\boldsymbol{u}^{n+1}, p^{n+1}, \boldsymbol{\lambda}^{n+1}\right) \in X \times M \times \mathcal{L}^{n+1}$ such that, for all $(\boldsymbol{v}, q, \mu) \in X \times M \times \mathcal{L}^{n+1}$,

$$
\begin{aligned}
\int_{\Omega} \rho \frac{\boldsymbol{u}^{n+1}-\boldsymbol{u}^{n}}{\delta t} \cdot \boldsymbol{v}+\int_{\Omega} \rho \boldsymbol{u}^{n} \cdot \nabla \boldsymbol{u}^{n+1} \cdot \boldsymbol{v}+\int_{\Omega} 2 \eta \mathbf{D}\left(\boldsymbol{u}^{n+1}\right): \mathbf{D}(\boldsymbol{v}) & \\
-\int_{\Omega} p^{n+1} \operatorname{div} \boldsymbol{v}+<\boldsymbol{\lambda}^{n+1}, \operatorname{Tr}_{\Sigma^{n+1}}(\boldsymbol{v})> & =-\int_{\Gamma_{i n}} p_{i n} \boldsymbol{n} \cdot \boldsymbol{v}, \\
\int_{\Omega} q \operatorname{div} \boldsymbol{u} & =0, \\
\left\langle\mu, \operatorname{Tr}_{\Sigma^{n+1}}\left(\boldsymbol{u}^{n+1}\right)\right\rangle & =\left\langle\mu, \boldsymbol{u}_{\Sigma^{n+1}}^{n+1}\right\rangle,
\end{aligned}
$$

where $\langle\cdot, \cdot\rangle$ denotes the duality pairing on $\left(H^{-1 / 2}\left(\Sigma^{n+1}\right)\right)^{2} \times\left(H^{1 / 2}\left(\Sigma^{n+1}\right)\right)^{2}$.

Remark 3.1. Taking into account the subdivision of $\Omega$ between the left hand side and the right hand side, respectively $\Omega^{-}$and $\Omega^{+}$(see Fig. 3), we have:

$$
\begin{array}{r}
\int_{\Omega} \rho \frac{\boldsymbol{u}^{n+1}-\boldsymbol{u}^{n}}{\delta t} \cdot \boldsymbol{v}+\int_{\Omega} \rho \boldsymbol{u}^{n} \cdot \nabla \boldsymbol{u}^{n+1} \cdot \boldsymbol{v}+\int_{\Omega \backslash \Sigma(t)} 2 \eta \mathbf{D}\left(\boldsymbol{u}^{n+1}\right): \mathbf{D}(\boldsymbol{v}) \\
\left.-\int_{\Omega \backslash \Sigma(t)} p^{n+1} \operatorname{div} \boldsymbol{v}+<\boldsymbol{\lambda}^{n+1}, \operatorname{Tr}_{\Sigma^{n+1}}(\boldsymbol{v})\right\rangle=-\int_{\Gamma_{\text {in }}} p_{i n} \boldsymbol{n} \cdot \boldsymbol{v} .
\end{array}
$$

Applying Green's formula in the integrals over $\Omega^{-}$and $\Omega^{+}$, and using the fact that $\left(\boldsymbol{u}^{n+1}, p^{n+1}\right)$ solves problem (9), we obtain $\boldsymbol{\lambda}^{n+1}=-\left(\boldsymbol{\sigma}^{n+1,-} \cdot \boldsymbol{n}^{-}+\boldsymbol{\sigma}^{n+1,+} \cdot \boldsymbol{n}^{+}\right)$. Thus, with notation (13),

$$
\lambda^{n+1}=\boldsymbol{f}_{\Sigma}^{n+1} .
$$

In other words, the Lagrange multiplier $\lambda^{n+1}$ corresponding to the constraint $\boldsymbol{u}^{n+1}=\boldsymbol{u}_{\Sigma^{n+1}}^{n+1}$ is nothing but the jump of the hydrodynamic stress through the valve.

\subsubsection{Finite element approximation}

The spaces $X$ and $M$ are approximated by the finite element spaces $X_{h}$ and $M_{h}$. The simulations presented in this paper have been performed with $\mathbb{P}_{1} / \mathbb{P}_{1}$ stabilized finite elements. We denote by $N_{F}$ the number of degrees of freedom for each component of the velocity in $X_{h}$.

The 1D structure being rigid and fixed in one point, one degree of freedom is sufficient to define its position. Nevertheless, from the fluid point of view, it is convenient to define $N_{\Sigma}$ discretization points $\left(\mathbf{x}_{i}^{n+1}\right)_{i=1, \ldots, N_{\Sigma}}$ on $\Sigma^{n+1}$. The approximation of the Lagrange multipliers space $\mathcal{L}^{n+1}$ can be done in various ways. In this work, we make the following choice:

$$
\mathcal{L}_{h}^{n+1}=\left\{\boldsymbol{\mu}_{h} \text { measure on } \Sigma^{n+1}, \boldsymbol{\mu}_{h}=\sum_{i=1}^{N_{\Sigma}} \boldsymbol{\mu}_{i} \delta\left(\mathbf{x}_{i}^{n+1}\right), \boldsymbol{\mu}_{i} \in \mathbb{R}^{2}\right\},
$$

where $\delta\left(\mathbf{x}_{i}^{n+1}\right)$ denotes the Dirac measure at point $\mathbf{x}_{i}^{n+1}$. Note that this approach is meaningful only after discretization (see $[12,16]$ ). For $\boldsymbol{\mu}_{h} \in \mathcal{L}_{h}$ and $\boldsymbol{v}_{h} \in X_{h}$,

$$
<\boldsymbol{\mu}_{h}, \operatorname{Tr}_{\Sigma^{n+1}}\left(\boldsymbol{v}_{h}\right)>=\sum_{i=1}^{N_{\Sigma}} \boldsymbol{\mu}_{i} \boldsymbol{v}_{h}\left(\mathbf{x}_{i}\right) .
$$

This quantity is well-defined as soon as $X_{h} \subset\left(\mathcal{C}^{0}(\Omega)\right)^{2}$, which is the case with the present choice of finite element space. 


\subsubsection{Load computation}

Although the only quantity required to solve the structure problem is the total momentum (12), it is convenient to adopt a more general viewpoint, proceeding as if the structure were elastic. In particular, this will allow in the future to use the same framework with more complicated structure models. We thus consider a 1D finite element mesh on $\Sigma^{n+1}$. For the sake of simplicity the nodes of this mesh are assumed to match the points $\left(\mathbf{x}_{i}\right)_{i=1 . . N_{\Sigma}}$. We then introduce a finite element basis $\left(\phi_{1}^{S}, \ldots, \phi_{N_{\Sigma}}^{S}\right)$ for the structure. We denote by $\boldsymbol{u}_{\Sigma^{n+1}, h}^{n+1}$ the discrete structure velocity, and by $U_{\Sigma} \in\left(\mathbb{R}^{N_{\Sigma}}\right)^{2}$ the vector of the components of $\boldsymbol{u}_{\Sigma^{n+1}, h}^{n+1}$ with respect to the basis $\left(\phi_{1}^{S}, \ldots, \phi_{N_{\Sigma}}^{S}\right)$. The load transmitted to a generic structure equation discretized on this basis has the form $F=\left(F_{1}^{(1)}, \ldots, F_{N_{\Sigma}}^{(1)}, F_{1}^{(2)}, \ldots, F_{N_{\Sigma}}^{(2)}\right) \in\left(\mathbb{R}^{N_{\Sigma}}\right)^{2}$, with

$$
F_{i}^{(k)}=\int_{\Sigma^{n+1}} \phi_{i}^{S} f_{\Sigma}^{n+1,(k)}, \quad k=1,2, \text { and } i=1, \ldots, N_{\Sigma},
$$

where $f_{\Sigma}^{n+1,(k)}$ denotes the component $k$ of the stress $\boldsymbol{f}_{\Sigma}^{n+1}$ defined in (13).

We now propose a method based on an energetic argument, adapted from [6] to our specific formulation, to easily obtain $F$ from the solution of (15)-(17).

The power exchanged at the interface "as seen by the structure" is

$$
\int_{\Sigma^{n+1}} \boldsymbol{u}_{\Sigma^{n+1}, h}^{n+1} \cdot \boldsymbol{f}_{\Sigma}^{n+1} d l=\sum_{k=1}^{2}\left(F^{(k)}, U_{\Sigma}^{(k)}\right),
$$

where $(\cdot, \cdot)$ is the euclidian scalar product on $\mathbb{R}^{N_{\Sigma}}$.

For $k=1,2$, we denote by $u_{h}^{(k)}$ and $\lambda_{h}^{(k)}$ the $k^{t h}$ components of the velocity $\boldsymbol{u}_{h}$ and the Lagrange multiplier $\boldsymbol{\lambda}_{h}$, respectively. We denote by $U^{(k)} \in \mathbb{R}^{N_{F}}$ the vector of components of $u_{h}^{(k)}$ on the velocity finite element basis, and by $\Lambda^{(k)} \in \mathbb{R}^{N_{\Sigma}}$ the vector of components of the Lagrange multiplier $\boldsymbol{\lambda}_{h}$ on the basis $\left(\delta\left(\mathbf{x}_{i}^{n+1}\right)\right)_{i=1 . . N_{\Sigma}}$. The power exchanged at the interface "as seen by the fluid" is

$$
<\boldsymbol{\lambda}_{h}, \operatorname{Tr}_{\Sigma^{n+1}}\left(\boldsymbol{u}_{h}\right)>=\sum_{k=1}^{2}\left(\Lambda^{(k)}, K U^{(k)}\right)
$$

where $(\cdot, \cdot)$ is the euclidian scalar product in $\mathbb{R}^{N_{\Sigma}}$ and $K \in \mathbb{R}^{N_{\Sigma} \times N_{F}}$ is the interpolation matrix of the structure nodes $\left(\mathbf{x}_{i}\right)_{i=1 . . N_{\Sigma}}$ on the fluid mesh. In view of (19), and in view of the fact that the nodes on the structure mesh coincide with the points $\mathbf{x}_{i}$, the algebraic counterpart of relation (17) reads

$$
K U^{(k)}=U_{\Sigma}^{(k)}, \quad k=1,2 .
$$

From (21) and (22), we deduce that the energy exchange between the fluid and the structure is well-balanced (no spurious energy is introduced) as soon as

$$
\left(F^{(k)}, U_{\Sigma}^{(k)}\right)=\left(\Lambda^{(k)}, K U^{(k)}\right), \quad k=1,2 .
$$

Since (24) must be valid for all $\boldsymbol{u}_{\Sigma}$, relations (23) and (24) give the relation

$$
F=\Lambda \text {. }
$$

We now consider the specific case of a rigid structure. Using the fact that $\sum_{i=1}^{N_{\Sigma}} \phi_{i}^{S}=1$ and using (20) and (25), we find that the total load on $\Sigma$ is given by $\sum_{i=1}^{N_{\Sigma}} \Lambda_{i}$. Moreover, if we assume to have for each $\mathbf{x} \in \Sigma^{n+1}$ 
the isoparametric representation (which holds for the $\mathbb{P}_{1}$ finite elements used in this work)

$$
\mathbf{x}=\sum_{i=1}^{N_{\Sigma}} \phi_{i}^{S}(\mathbf{x}) \mathbf{x}_{i}
$$

the total momentum is given by:

$$
\mathcal{M}_{\Sigma^{n+1}}^{n+1}=\int_{\Sigma^{n+1}} \mathbf{C x} \times \boldsymbol{f}_{\Sigma}^{n+1} d l(\mathbf{x})=\sum_{i=1}^{N_{\Sigma}}\left(\mathbf{x}_{i}-\mathbf{C}\right) \times \int_{\Sigma^{n+1}} \phi_{i}^{S}(\mathbf{x}) \boldsymbol{f}^{n+1} d l(\mathbf{x})=\sum_{i=1}^{N_{\Sigma}}\left(\mathbf{x}_{i}-\mathbf{C}\right) \times \Lambda_{i} .
$$

\subsection{Energetic considerations}

In view of the discussion of the energy loss index of the next section as a diagnostic tool, we now draw up an energetic balance of our discrete fluid-valve interaction model. We follow the same procedure as in subsection 2.4 for the continuous model; we thus obtain:

$\frac{E_{k, h}^{n+1}-E_{k, h}^{n}}{\delta t}+P_{v i s c, h}^{n+1}+P_{v a l v e, h}^{n+1}+P_{n u m, h}^{n+1}=\int_{\Gamma_{\text {in }}}\left(p_{h}^{n+1}+\frac{\rho}{2}\left(\boldsymbol{u}_{h}^{n+1}\right)^{2}\right) \boldsymbol{u}_{h}^{n+1} \cdot \boldsymbol{e}_{x}-\int_{\Gamma_{\text {out }}}\left(p_{h}^{n+1}+\frac{\rho}{2}\left(\boldsymbol{u}_{h}^{n+1}\right)^{2}\right) \boldsymbol{u}_{h}^{n+1} \cdot \boldsymbol{e}_{x}$,

with

$$
E_{k}^{n+1}=\frac{1}{2} \int_{\Omega} \rho\left(\boldsymbol{u}^{n+1}\right)^{2}, \quad P_{v i s c, h}^{n+1}=\int_{\Omega} 2 \eta\left|D\left(\boldsymbol{u}^{n+1}\right)\right|^{2}, \quad P_{\text {valve }, h}^{n+1}=\int_{\Sigma} \boldsymbol{f}_{\Sigma}^{n+1} \cdot \boldsymbol{u}^{n+1},
$$

and where $P_{n u m, h}^{n+1}$ denotes the numerical diffusion induced by the discretization. In Sect. 5 , the quantities $P_{v i s c, h}^{n+1}$ and $P_{v a l v e, h}^{n+1}$ will be monitored in different stenotic configurations.

\section{Clinical assessment of stenosis}

In order to guide clinicians in their diagnosis of the aortic valve stenosis, standard parameters are at their disposal (see [2] for a general survey of this topic). These clinical indexes are based on velocity and pressure measurements and are realized by catheterization or, more often, by Doppler echocardiography which presents the advantage to be a non-invasive procedure. These clinical indexes have been established from simplified fluid mechanics equations.

On the one hand, mean pressure difference $\left(\overline{T P G}=\overline{p_{V}-p_{V C}}\right)$ is assessed between the left ventricle (V) and the vena contracta (VC), where the cross-sectional area of the flow jet is minimal (see Fig. 4). Using catheterization, this measure is direct. Using Doppler, this value is obtained from the measure of the upstream velocity $V_{V}$ and the transvalvular flow velocity $V_{V C}$. Indeed, applied on a streamline between the left ventricle and the vena contracta, neglecting the gravity potential, knowing that there is no energy losses between the two sections and using a temporal mean, the Bernoulli equation yields

$$
\overline{T P G}=\overline{p_{V}-p_{V C}}=\frac{1}{2} \rho\left(\overline{V_{V C}^{2}}-\overline{V_{V}^{2}}\right) .
$$

Clinicians generally make the assumption that the ventricular velocity $V_{V}$ can be neglected. Moreover, they typically used $m m H g$ as pressure unit and a value $\rho \approx 1000 \mathrm{~kg} / \mathrm{m}^{3}$ in blood, this equation is reduced to:

$$
\overline{T P G}=\overline{p_{V}-p_{V C}}=4\left(\overline{V_{V C}^{2}}\right) .
$$

A mean pressure difference that exceeds $20 \mathrm{mmHg}$ is considered significant and when $\overline{T P G}>50 \mathrm{mmHg}$, the stenosis is classified severe. 


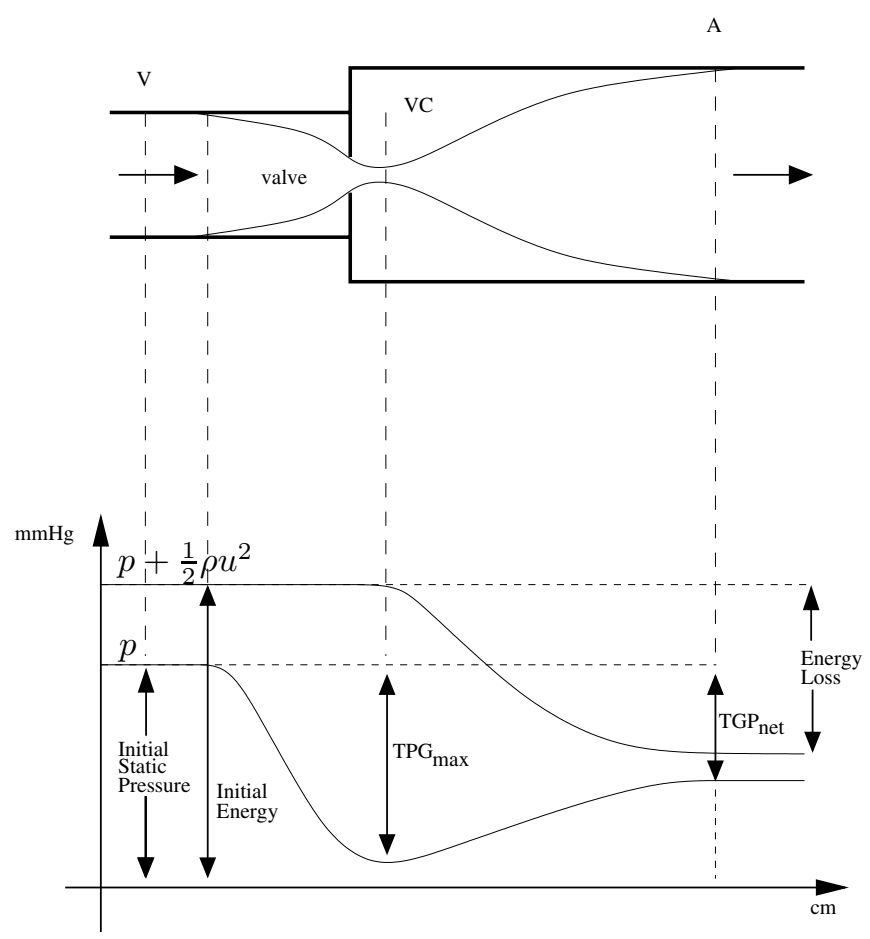

FIGURE 4. Top: a simplified sketch of the aortic valve geometry. The left heart ventricle is on the left side, while the aorta is on the right. Bottom: dynamic and static pressure profiles along the axis. Observe the pressure drop and then the subsequent partial recovery (reproduced from [10]).

On the other hand, clinicians can estimate the so-called aortic valve Effective Orifice Area (EOA), which is the minimal cross-sectional area of the flow jet, attained downstream the valve at the vena contracta. Using Doppler, this value is obtained from the application of the continuity equation between the left ventricle and the vena contracta. Assuming that the values of the areas are not modified during systolic ejection, that the section of the ventricle is circular with a diameter $A_{V}$ and that velocity profiles are flat, EOA is expressed in $\mathrm{cm}^{2}$ as:

$$
\mathrm{EOA}_{\text {Doppler }}=\frac{1}{v_{V C}}\left(\pi\left(\frac{A_{V}}{2}\right)^{2} v_{V}\right),
$$

Using catheterization, EOA is evaluated from the Gorlin equation (see [14]) and is expressed in $\mathrm{cm}^{2}$ :

$$
\mathrm{EOA}_{\mathrm{Gorlin}}=\frac{\bar{Q}}{44.3 \sqrt{\overline{T P G}}}
$$

where $Q$ is the flow rate expressed in $m L / s$ and $\overline{T P G}$ unit is $m m H g$. The value 44.3 is an empirical factor that derived from the original equation proposed by Gorlin (see [14]).

The grade of the aortic stenosis severity depends on the value of EOA: mild (EOA $\left.>1.5 \mathrm{~cm}^{2}\right)$, moderate $\left(1<\mathrm{EOA} \leq 1.5 \mathrm{~cm}^{2}\right)$ and severe $\left(\mathrm{EOA} \leq 1 \mathrm{~cm}^{2}\right)$. Although all these measures should lead to the same value, it has been recognized (see for example [9]) that Doppler- and catheter-derived valve EOAs are often discrepant, resulting in potentially divergent estimations of aortic valve severity. These discrepancies are largely due to the pressure recovery phenomenon that occurs downstream the valve due to the conversion of a certain amount 
of kinetic energy (dynamic pressure) to static pressure (see [3] and [4]). Indeed, whilst Doppler measures the velocity in the VC section, it is generally very difficult to obtain the correct $\overline{T P G}$ by catheter because of the difficulty in adjusting and maintaining the position of the pressure sensor. Then instead, a measurement of the net pressure gradient $T P G_{n e t}$ is often obtained (see Fig. 4 bottom).

The EOA measurement is currently used to assess the degree of stenosis severity and is recommended by the American Heart Association (AHA) and the American College of Cardiology (ACC). Nevertheless, from a mechanical point of view, the presence of a stenosis can be considered as a resistance to blood flow. As a consequence, a part of the energy supplied by the ventricle work is dissipated. Assuming that this loss is more relevant than the EOA, a new diagnostic parameter has been recently proposed in [10]. From the Bernoulli equation applied on a streamline between the left ventricle and the aorta, considering that energy losses exist only between the vena contracta and the aorta and using the continuity equation and the momentum equation between the sections including the vena contracta and the aorta, the energy loss $E_{L}$ can be expressed as:

$$
\overline{E_{L}}=\frac{\rho}{2} \overline{V_{V C}^{2}}\left(1-\frac{\mathrm{EOA}}{A_{A}}\right)^{2}
$$

where $A_{A}$ is the aorta section (assumed to be constant like EOA).

As $\bar{Q}=\overline{V_{V C}}$.EOA and to express $E_{L}$ in $m m H g$, following simplifications can be made to be easily used:

$$
\overline{E_{L}}=4 \overline{Q^{2}}\left(\frac{1}{\mathrm{EOA}}-\frac{1}{A_{A}}\right)^{2} .
$$

The units are $m L / s$ for $\bar{Q}$ and $\mathrm{cm}^{2}$ for EOA and $A_{A}$. From (26), the new proposed index is then:

$$
\frac{\mathrm{EOA} \cdot A_{A}}{A_{A}-\mathrm{EOA}} \text {. }
$$

Its main advantage is to be easily obtained by Doppler and seems to be a better predictor of outcomes than the classical EOA since it describes a more physical quantity directly linked to damage caused by the stenosis on the heart work.

\section{Numerical RESUlts}

In this section we present different simulations carried out to validate the numerical procedure and to investigate the energy dissipation.

In each test case, the pressure on $\Gamma_{i n}$ is defined by the periodic function:

$$
p_{\text {in }}(t)= \begin{cases}p_{0}, & 0 \leq t<T_{1} \\ \left(-\left(t-T_{1}\right) p_{0}+\left(T_{2}-t\right) p_{0}\right) /\left(T_{2}-T_{1}\right), & T_{1} \leq t<T_{2} \\ -p_{0}, & T_{2} \leq t<T_{3} \\ \left(\left(t-T_{3}\right) p_{0}-\left(T_{4}-t\right) p_{0}\right) /\left(T_{4}-T_{3}\right), & T_{3} \leq t<T_{4}\end{cases}
$$

The values for $T_{1}, T_{2}, T_{3}, T_{4}$ are respectively $0.3,0.4,0.7,0.8$, and $p_{0}=500$. This pressure function is represented on Fig. 5, top left. Over $\Gamma_{\text {out }}$, the pressure is constant and equal to zero. The other physical parameters are: $J=0.51, \rho=1, \eta=0.03$. The length of the valve is 0.8 whereas the width of the tube is 1 (as a consequence, the valve cannot close the pipe). 


\subsection{Test 1}

In this test case we consider a straight tube and we monitor the power dissipated by viscous effects. At $t=0$, the angle $\theta$ is 90 degrees (see Fig. 3 for the orientation) and the fluid is at rest. The maximum value of $\theta$ is forced to be less than 90 degrees. The minimum value is forced to be greater than 10, 20 or 45 degrees, depending on the test case. These three different "blocking angles" are supposed to model three levels of stenosis: the larger the blocking angle is, the stronger is the stenosis. In this preliminary work, the valve blocking is enforced via a simple test on the value of the angle. The results are represented on Fig. 5 (bottom right). This result confirms the fact that a strongest stenosis induces a largest energy loss through the valve.

On Fig. 6 we represent the static $(p)$ and the dynamic $\left(p+\rho \boldsymbol{u}^{2} / 2\right)$ pressures at the end of an opening period for the strongly stenotic case (blocking angle of $45^{\circ}$ ). This result is in good agreement with the qualitative behaviour described in [10] and reproduced in Fig. 4.
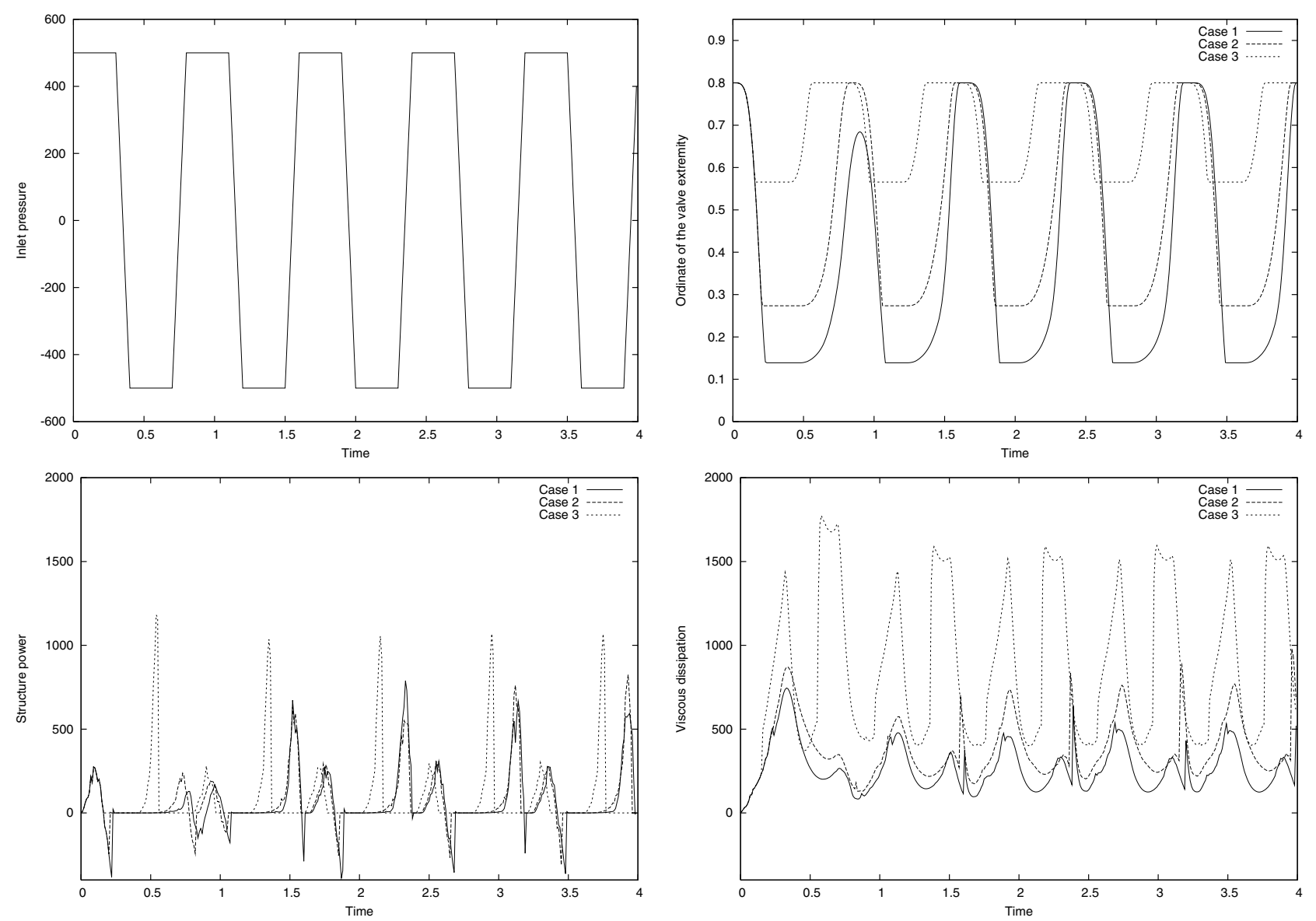

Figure 5. Simulations on a straight $2 \mathrm{D}$ pipe with different maximum valve openings. Case 1 : $10^{\circ}$ (smallest stenosis), case 2: $20^{\circ}$, case 3: $45^{\circ}$ (strongest stenosis). Top left : inlet pressure vs. time. Top right : ordinate of the extremity of the valve vs. time. Bottom left: structure power $\left(P_{\text {valve }, h}\right)$. Bottom right: viscous dissipation $\left(P_{v i s c, h}\right)$. As expected, the strongest stenosis gives the maximum energy loss. 


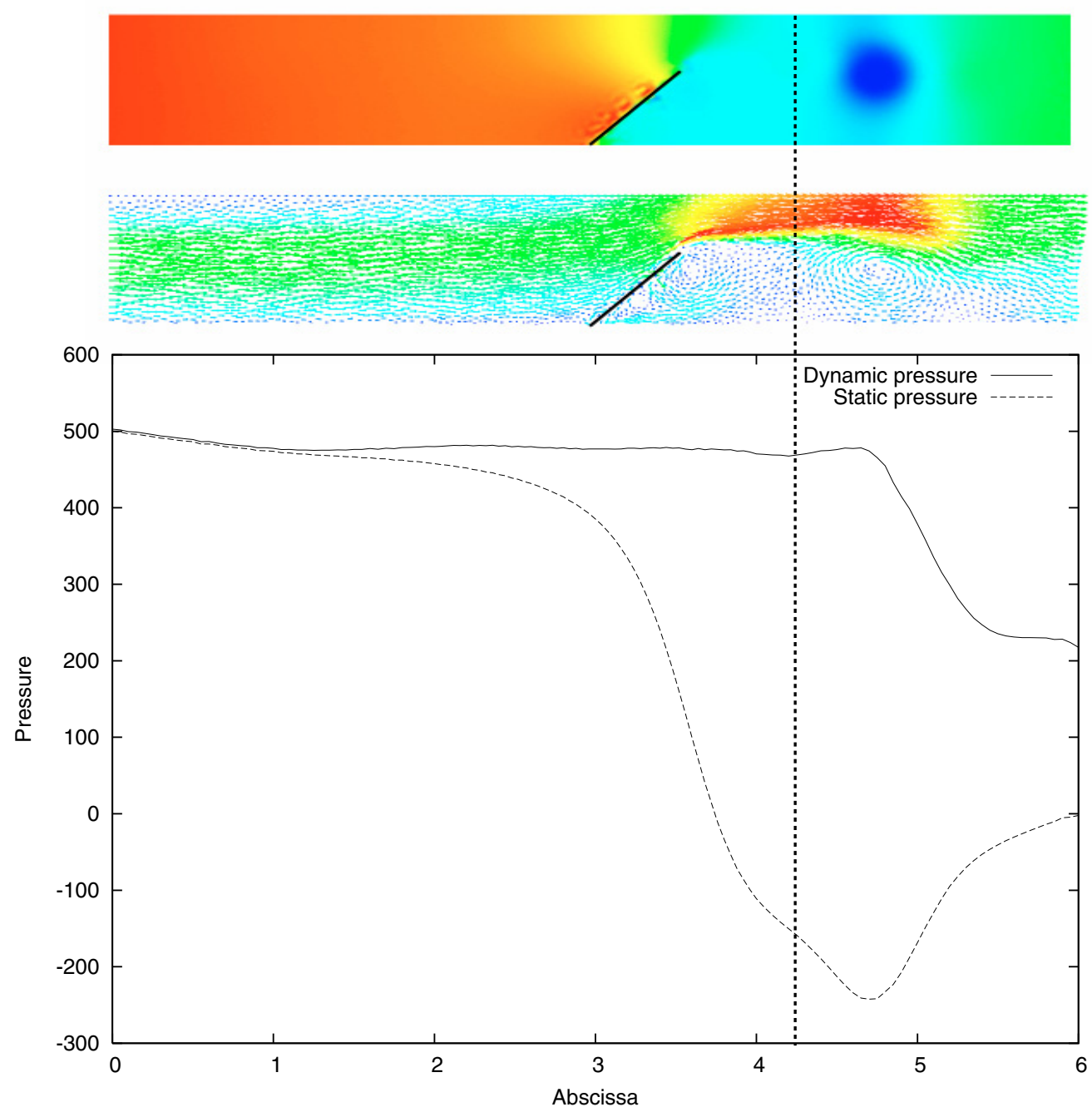

FIGURE 6. Top: snapshot of the pressure and the velocity fields at time $t=2.67$ corresponding to the end of a maximum opening period in the Case 3 (strongest stenosis) of Fig. 5. Bottom: corresponding static pressure $(p)$ and dynamic pressure $\left(p+\frac{\rho}{2} \boldsymbol{u}^{2}\right)$ along the axis. The vertical dashed line indicates approximatively the position of the vena contracta. Qualitatively, the static and the dynamic pressures are in good agreement with the results reported in [10] and reproduced in Fig. 4.

\subsection{Test 2}

The geometry considered in this test case is supposed to roughly mimic the presence of an aortic sinus, a physiological cavity in the aorta root. The purpose of this simulation is purely illustrative. Figures 7 and 8 show an example of velocity and pressure fields in the case of the strongest stenosis (blocking angle of 45 degrees) for two different times. 


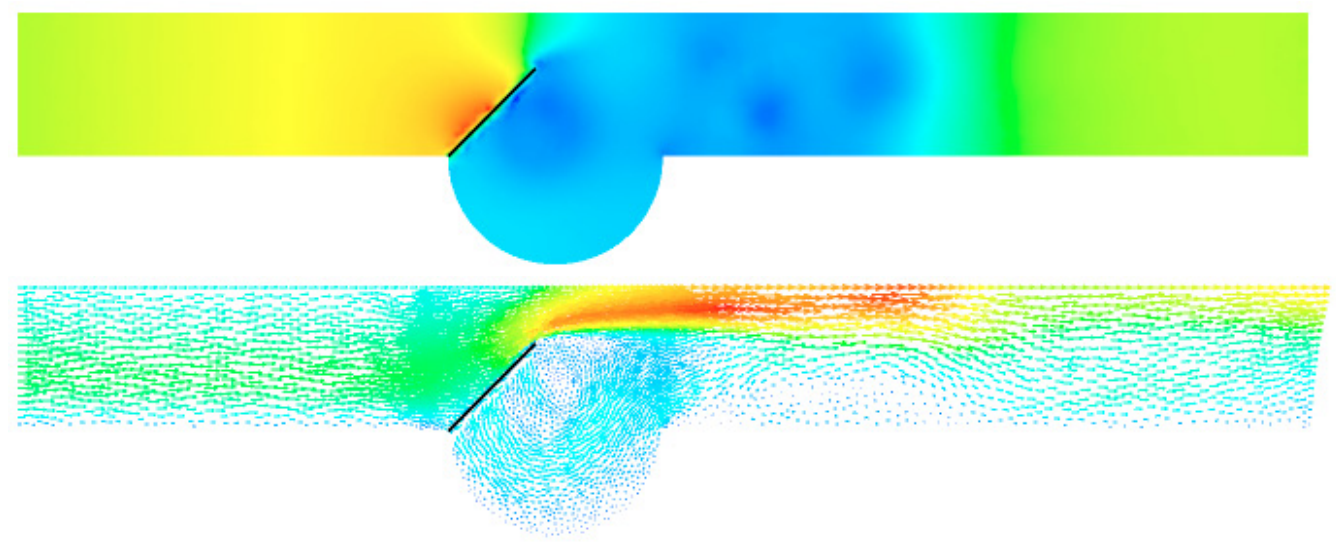

Figure 7. Pressure and velocity fields at $t=2.65$ (maximum opening in a stenotic case).

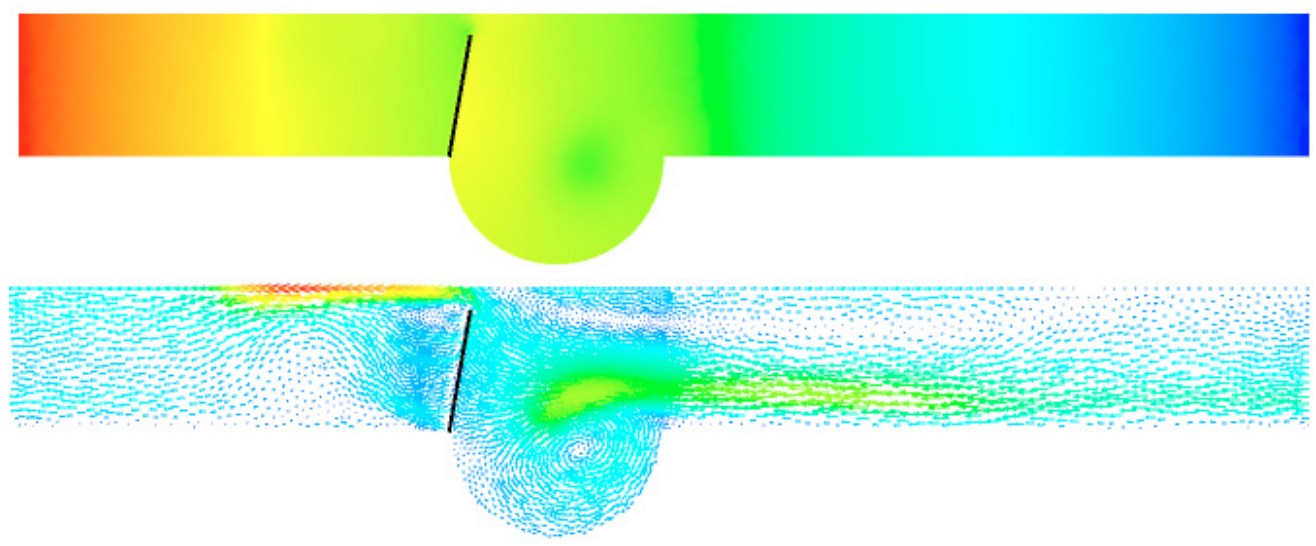

Figure 8. Pressure and velocity fields at $t=3.05$ (end of a closing period). The "regurgitation" is due to the fact that in this simplified model the valve does not close completely the pipe.

\section{Conclusions And Perspective}

In this work, we have proposed a numerical framework for the simulation of the complex behaviour of the aortic valve. This work does not address all the complexity of the behaviour of the valve, for example valve complete closure is not allowed and the structure model is rather simple, but it paves the way for more sophisticated models. In particular it aims to become a tool for evaluating the physical correctness of the synthetic indexes used in the medical practice. Among the important aspects to be dealt with in the future, we cite the coupling with the arterial wall movement, that is known to affect the valve behaviour.

\section{ACKNOWLEDGEMENTS}

We thank Bertrand Maury and Valérie Deplano for helpful discussions. 


\section{REFERENCES}

[1] F. Baaijens. A fictitious domain/mortar element method for fluid-structure interaction. Int. Jour. Num. Meth. Fluids, 35:743$761,2001$.

[2] R.O. Bonow, B.A. Carabello, A.C. de Leon, L.H. Edmunds, B.J. Fredderly, M.D. Freed, W.H. Gaasch, R.A. McKay, C.R.and Nishimura, P.T. O'Gara, R.A. O'Rourke, and S.H. Rahimtoola. Guidelines for the management of patients with valvular heart disease: executive summary, a report of the american college of cardiology. Circulation, 98:1949-1984, 1998.

[3] C. Clark. The fluid mechanics of the aortic stenosis - I. Theory and steady flow experiments. J. Biomech., 9:521-528, 1976.

[4] C. Clark. The fluid mechanics of the aortic stenosis - II. Unsteady flow experiments. J. Biomech., 9:567-573, 1976.

[5] J. De Hart, G.W.M. Peters, P.J.G. Schreurs, and F.P.T. Baaijens. A three-dimensional computational analysis of fluid-structure interaction in the aortic valve. Journal of Biomechanics, 36:103-112, 2003.

[6] C. Farhat, M. Lesoinne, and P. Le Tallec. Load and motion transfer algorithms for fluid/structure interaction problems with non-matching discrete interfaces: Momentum and energy conservation, optimal discretization and application to aeroelasticity. Comput. Methods Appl. Mech. Engrg., 157:95-114, 1998.

[7] M. Fernández, A. Moura, and C. Vergara. Defective boundary conditions applied to multiscale analysis of blood flow. in this proceedings.

[8] L. Formaggia, J.-F. Gerbeau, F. Nobile, and A. Quarteroni. Numerical treatment of defective boundary conditions for the Navier-Stokes equations. SIAM J. Numerical Analysis, 40(1):376-401, 2002.

[9] D. Garcia, J. Dumesnil, L.-G. Durand, L. Kadem, and P. Pibarot. Discrepancies between catheter and doppler estimates of valve effective orifice area can be predicted from the pressure recovery phenomenon. Jour. American College of Cardiology, 41(3):435-445, 2003.

[10] D. Garcia, P. Pibarot, J. Dumesnil, F. Sakr, and L.-G. Durand. Assessment of aortic valve stenosis severity, a new index based on the energy loss concept. Circulation, 101:765-771, 2000.

[11] J.-F. Gerbeau and M. Vidrascu. A quasi-Newton algorithm based on a reduced model for fluid-structure interactions problems in blood flows. Math. Model. Num. Anal., 37(4):631-648, 2003.

[12] R. Glowinski, T.W. Pan, T.I. Hesla, and D.D. Joseph. A distributed Lagrange mutiplier/fictitious domain method for particulate flows. Int. J. of Multiphase Flow, 25:755-794, 1999.

[13] R. Glowinski, T.W. Pan, and J. Périaux. A fictitious domain method for Dirichlet problem and applications. Comput. Methods Appl. Mech. Engrg., 111(3-4):283-303, 1994.

[14] R. Gorlin and S.C. Gorlin. Hydraulic formula for calculation of the area of the stenotic mitral valve, other cardiac valves, and central circulatory shunts. Am. Heart J., 41:1-29, 1951.

[15] http://www.nlm.nih.gov/medlineplus. A service of the U.S. National Library of medicine and the National Institutes of Health and the National Institutes of Health.

[16] L. H. Juárez, R. Glowinski, and T. W. Pan. Numerical simulation of the sedimentation of rigid bodies in an incompressible viscous fluid by Lagrange multiplier/fictitious domain methods combined with the Taylor-Hood finite element approximation. In Proceedings of the Fifth International Conference on Spectral and High Order Methods (ICOSAHOM-01) (Uppsala), volume 17, pages 683-694, 2002.

[17] B. Maury. Direct simulations of 2D fluid-particle flows in biperiodic domains. J. Comput. Phys., 156(2):325-351, 1999.

[18] D. P. Mok, W. A. Wall, and E. Ramm. Accelerated iterative substructuring schemes for instationary fluid-structure interaction. In K.J. Bathe, editor, Computational Fluid and Solid Mechanics, pages 1325-1328. Elsevier, 2001. 